\title{
ADAPTIVE CORRECTION FOR ACOUSTIC IMAGING IN DIFFICULT MATERIALS
}

\author{
I. J. Collison, S. D. Sharples, M. Clark and M. G. Somekh \\ Applied Optics, Electrical and Electronic Engineering, University of Nottingham, \\ University Park, Nottingham, UK, NG7 2RD
}

\begin{abstract}
The technological developments in the design of an adaptive optical scanning acoustic microscope are described. Its two key elements include a multi-channel acoustic wavefront detector to detect the acoustic wavefront aberration and a highly adaptive acoustic source to correct for the effects of aberration. We present experimental images acquired with the instrument that indicate the degree of performance improvement achieved when adaption is used to correct for material aberration.
\end{abstract}

\section{INTRODUCTION}

Acoustic waves travelling in heterogeneous materials (such as polycrystalline metals) can be aberrated (or distorted) by the micro-structure. This occurs because different parts of the wave 'see' different velocities which distorts the wavefront. The effect becomes more severe as the wavelength approaches a similar order of magnitude to the size of the microstructure. This effect is distinct from scattering as it primarily distorts the passage of the forward travelling wave. This aberration can result in the wave moving from its intended position or the distribution of the energy of the wave breaking up into speckles. Both of these effects can severely degrade the performance of acoustic imaging techniques. The effects become more severe at higher frequencies, longer path lengths and larger grain sizes in metals.

We have constructed an adaptive ultrasound system which can compensate for the effects of aberrating materials and maintain good image quality at higher frequencies, longer path lengths and through larger grain sizes than previously possible. This optical-scanningacoustic-microscope (O-SAM) uses a novel acoustic wavefront sensor (AWFS) and an adaptive optical source to detect the distortions caused by the aberrating grain structure and compensate for them.

In this paper we present two techniques that are used for aberration correction. The first is simple source tilt correction which simply redirects the acoustic energy back to its intended location. The second is a higher order technique which not only redirects the acoustic energy but also attempts to reconstruct the original distribution.

\section{OPTICAL SCANNING ACOUSTIC MICROSCOPE}

The O-SAM instrument [1] is shown schematically in figure 1. The generation laser is a Q-switched, mode-locked Nd:YAG laser. A spatial light modulator (SLM) is used to generate a distribution of concentric arcs which are imaged onto the sample. The fringe spacing of the 


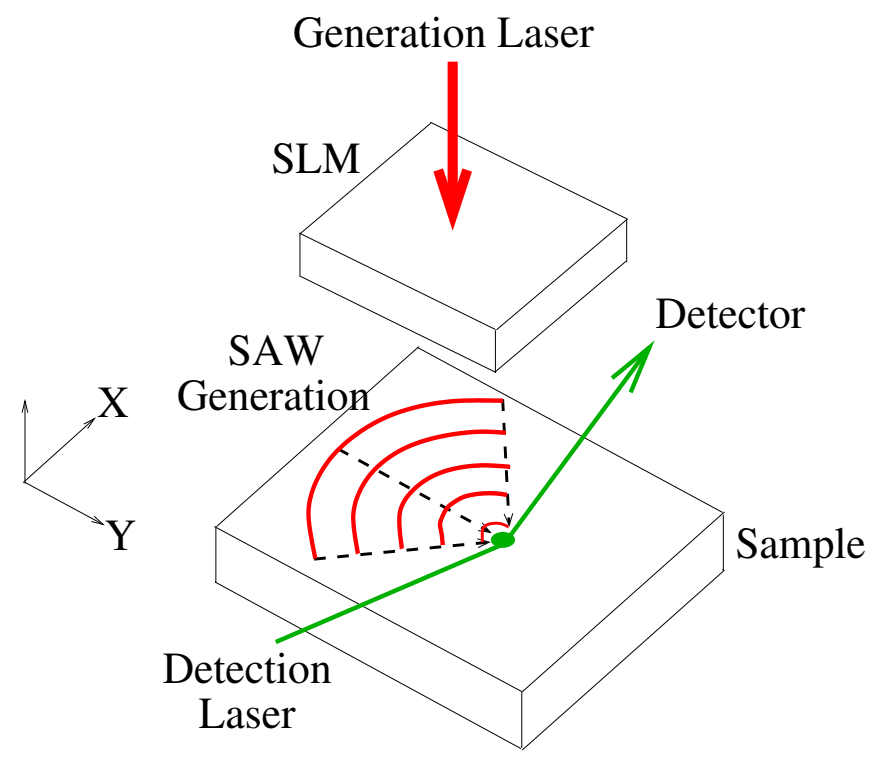

FIGURE 1. Schematic diagram of the O-SAM instrument. The light from the generation laser is imaged onto the sample by the SLM. The detector is a modified knife-edge design using a split photodiode.

arcs corresponds to the wavelength of the acoustic waves on the material. The advantages of the SLM are that the generation profile can be changed electronically thus controlling the generated ultrasound like a phased array and the SLM distributes the light over a large area, eliminating damage.

The detection system consists of a doubled Nd:YAG laser and a modified knife-edged detector in which the knife-edge is replaced by a split photodiode. As the surface acoustic waves (SAW) pass under the detection probe, the reflected light oscillates between the two detectors in the split photodiode and the amplitude and phase of the acoustic waves are measured. By scanning the sample on XY stages and keeping a constant relative position between the generation and detection regions, c-scan images are obtained. Another useful feature of the O-SAM is that by keeping the sample in a fixed position relative to the generation region and scanning the detection probe, aerial point spread functions (PSF) can be measured.

\section{EFFECTS OF ABERRATION}

Figure 2 compares the effects that isotropic and aberrating media have on wavefront propagation. The aerial PSFs show the amplitude of the surface waves as they propagate from the excitation to the detection region. On all the images in figure 2 the detection point has been marked. On isotropic media, the focused SAWs generated by the SLM maintain their shape as they propagate and come to a focus at the intended detection point. On the aberrating medium, the surface waves have missed the detection point and the acoustic energy has also dispersed due to the random grain structure of the material.

In order to correct for material aberrations, it is necessary to collect information about the acoustic wavefront around the detection point. Before the introduction of the AWFS into the O-SAM system, this was achieved using a single detector which was linearly scanned to get this information. With the AWFS experimental times have been reduced by approximately 2 orders of magnitude by using multiple detectors simultaneously. 

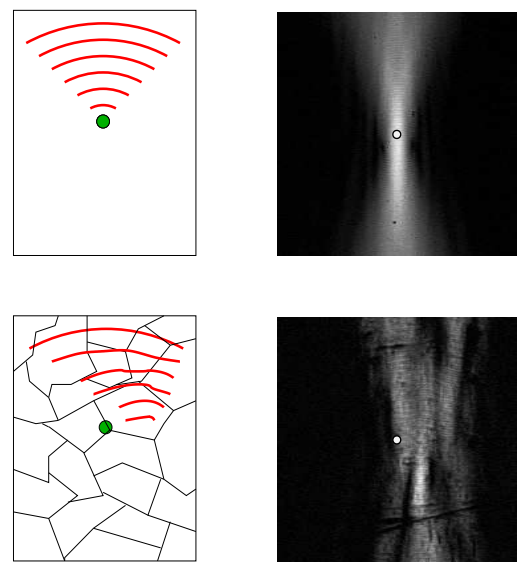

FIGURE 2. Images on the left hand side show schematically the propagation of SAWs on a uniform and isotropic media (above) and a random aberrating media (below). On the right, aerial PSFs taken on aluminumcoated glass (above) and aluminum (below).

\section{ACOUSTIC WAVEFRONT SENSOR}

The integration of the AWFS into the OSAM system is at an early stage and although the full bandwidth of the device has not yet been determined, it is possible to detect acoustic waves at a frequency of $328 \mathrm{MHz}$ [2]. The AWFS is a custom silicon photodiode array built using a standard CMOS (silicon chip) process. It consists of an array of 16 split photodiodes. In order to detect at 16 locations around the focal region simultaneously, an astigmatic optic is used to spread the detection point into a detection line on the sample. This line is imaged onto the photodiode array and forms a $16 \times 1$ knife edge detector array which detects the acoustic aberrations. The acquired information is then used to estimate a new generation profile which will compensate for the aberrations. The SLM is then reprogrammed with this corrected generation profile and an improved ultrasound distribution is generated and used to take measurements. Using the AWFS this entire procedure can be achieved at up to 100 times a second. Figure 3 is a schematic representation showing the role that the SLM and AWFS have within the O-SAM system.

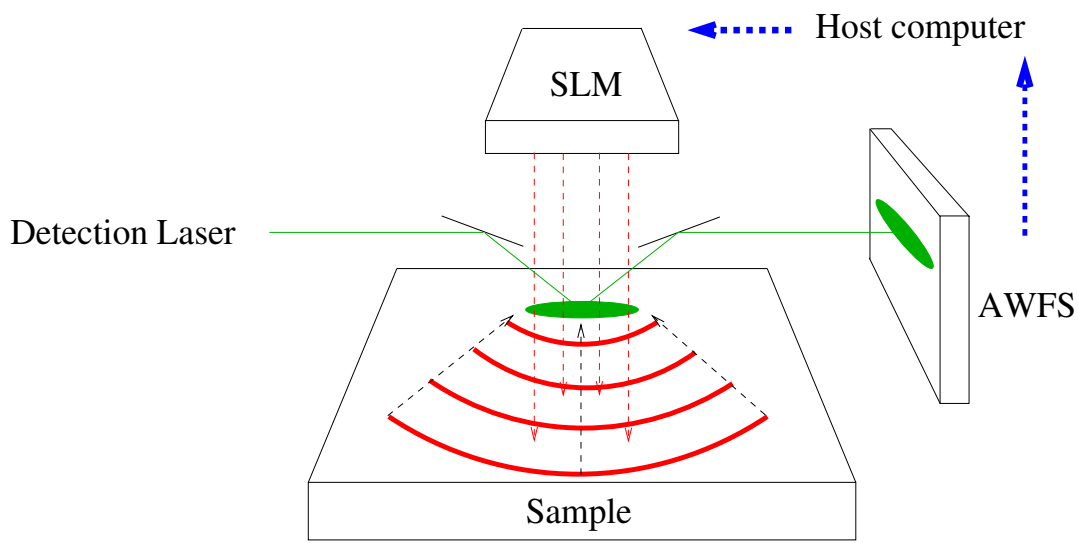

FIGURE 3. The system used for high speed SAW detection and higher order aberration correction. The aberrations are detected with the AWFS and used to produce a new distribution for the SLM which improves the ultrasound distribution, via the host computer in the feedback path. 

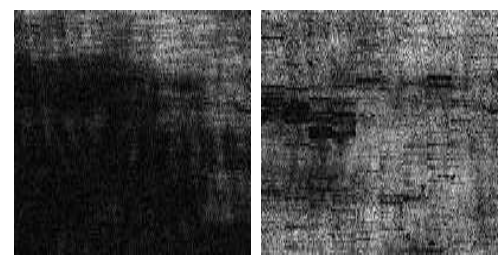

FIGURE 4. Amplitude c-scan images before (left) and after (right) tilt correction. Image size is $5 \mathrm{~mm} \times 5 \mathrm{~mm}$.

\section{SOURCE TILT CORRECTION}

When the aberration is low order the acoustic energy will just be deviated from side to side. In this case simple 'source tilt' correction can be used to steer the acoustic energy back to the measurement region. This is achieved by detecting the deviation of the ultrasound using the AWFS and applying an equal but opposite tilt to the generation profile. Figure 4 shows results before and after source tilt correction on a strongly aberrating aluminum sample. Before correction much of the signal has been degraded and lost because of low order aberration. This can be seen by the large dark region in the left hand image of figure 4 . Having applied source tilt correction, much of the signal has been recovered as shown in the right hand image. However in some regions source tilt correction is not sufficient to recover all of the signal. This can be seen where dark regions still remain and in these areas higher order correction is needed.

This experiment was carried out prior to the installation of the AWFS and in this case the aberrations were detected by scanning a single point. As a result the data for figure 4 took four hours to acquire.

\section{HIGHER ORDER ABERRATION CORRECTION TECHNIQUE}

A simple first order correction technique such as source tilt correction is viable when the effects of aberration are low order (i.e. contains low spatial frequencies only). However, materials which cause higher order aberrations, lead to the acoustic wavefront dispersing and breaking up. In this case a higher order correction technique is required.

In order to do this, the aberrations are detected with the AWFS which is then used to compute a new generation profile to compensate for the aberrations caused by the material [3]. This process is shown schematically in figure 5(a). The acoustic amplitude at the measurement region before and after correction is shown in figure 5(b). This demonstrates that the higher order algorithm not only recenters the ultrasound but also refocuses it through the

(a)
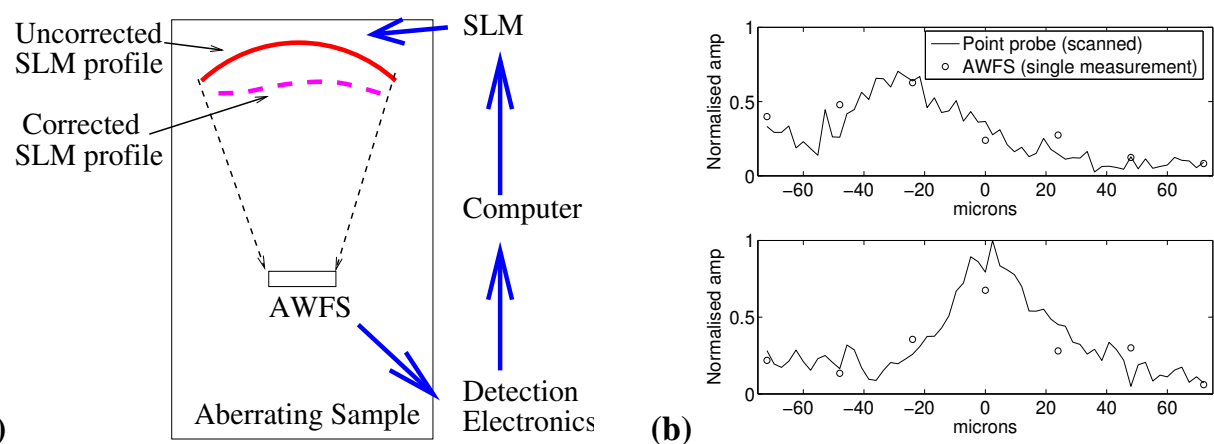

FIGURE 5. The higher order correction process (a) and the amplitude response measured by AWFS before and after correction (b). In both plots, the solid line is the amplitude obtained by scanning a single detector of the AWFS along the detection region. The single points are the amplitudes measured by 7 detectors. 
aberrating material.

The error resulting from the aberration is estimated at the generation region by back propagating the wavefront detected by the AWFS using an angular spectrum technique [4]. This error is then combined with the ideal generation profile to produce a 'corrected' generation profile. The new corrected profile is then downloaded onto the SLM and used to generate an acoustic wavefront which compensates for the aberration and produces an improved ultrasound distribution.

\section{PRELIMINARY RESULTS USING HIGHER ORDER ABERRATION CORREC- TION}

A useful tool for comparing correction techniques is to construct w-scans. These are a direct indication of PSF quality as the sample is moved. Figure 6 shows three w-scans. These were constructed by scanning along $2 \mathrm{~mm}$ of large grained $(600 \mu \mathrm{m} \times 200 \mu \mathrm{m})$ aluminum. At every point along the scan line, the complex amplitude of the PSF was obtained. This data was then used to generate a single plot which shows the PSFs 'wandering off' from the central position i.e. a wander scan.

The top image is the uncorrected w-scan, the middle one shows the result of applying source tilt correction only and the bottom image shows higher order correction. To the right of the w-scans, cross-sectional amplitude plots are shown, the location of which is indicated by the vertical dashed line on the w-scans. These show the degree of 'acoustic wander' from the intended line of travel or the zero deviation line.

Clearly with the uncorrected w-scan, the material aberrations have caused the acoustic energy to wander and break up into multiple sidelobes. This is indicated by the amplitude graph on the right. By applying tilt correction, the acoustic energy has been steered back to the detection point but it is still broken and attenuated because it is still spread out. With higher order correction, not only has the acoustic energy maintained its correct line of travel, but has also been refocused to give a higher and more narrow amplitude response.

$\mathrm{C}$-scans can also be used to indicate the degree of acoustic image improvement. Figure 7 shows preliminary results taken with the prototype adaptive O-SAM instrument. The amplitude and phase responses without and with higher order correction using 7 channels of

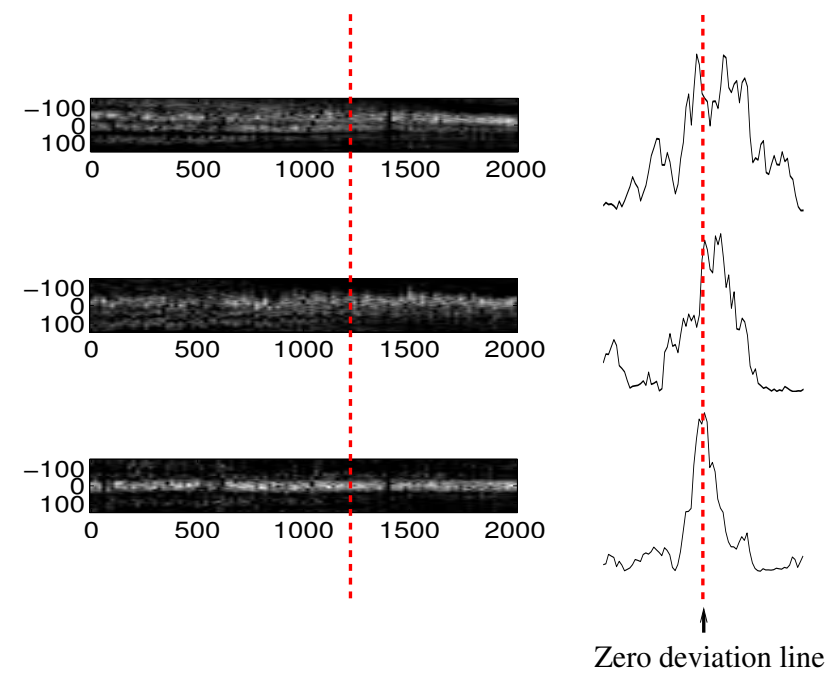

FIGURE 6. Wander scans before correction (top), after tilt correction (middle) and after higher order correction (bottom). Corresponding amplitudes of a cross-section indicated by the vertical line are on the right. Image size is $0.3 \mathrm{~mm} \times 2 \mathrm{~mm}$. 


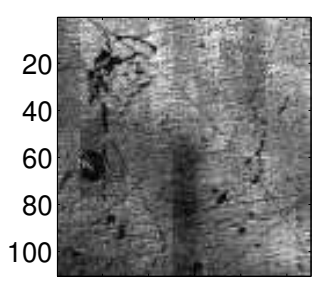

20406080100

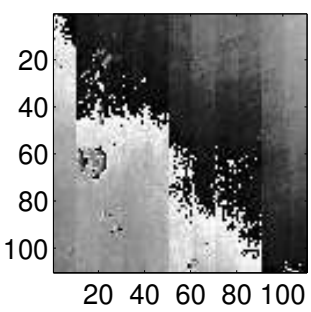

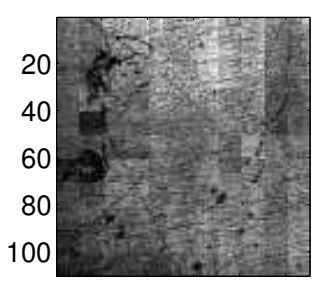

20406080100

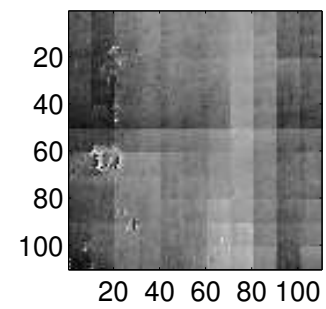

FIGURE 7. Amplitude (above) and phase (below) c-scans at $82 \mathrm{MHz}$ on aluminum before (left) and after (right) higher order correction using 7 detectors of the AWFS. In order to facilitate correction with the prototype O-SAM, the image was made up of discrete patches. These can be eliminated by performing the correction algorithm automatically at a speed of 100 points/second.

the AWFS are shown. In this preliminary case a mildly aberrating aluminum sample was used as a test piece for this experiment. The sample used did not have any defects and so uniform amplitude and phase images would be expected. However, images on the left of figure 7 show that the material microstructure has caused acoustic aberration. Having applied higher order correction, more uniform images have been obtained, in particular with the phase response. This is shown on the right of figure 7.

\section{CONCLUSIONS AND FURTHER WORK}

Source tilt correction is limited because it can only correct for slightly aberrating materials. For difficult materials where there is a higher degree of aberration, a first order correction technique is insufficient and therefore a higher order method must be employed. Before the installation of the AWFS into the O-SAM system, information about the propagating wavefronts was gathered by scanning a single detector across the focus of the PSF. This paper has shown that higher order correction can be successfully implemented by simultaneously detecting the acoustic wavefront at several discrete points with the AWFS. Only minor alterations to the scanning procedure have had to be made in order to incorporate the device into the imaging process. Work is in progress to integrate the AWFS and the detection electronics onto a single chip to form an integrated optical sensor. This would significantly reduce complexity, cost and the variability of a multi-channel detector system.

\section{REFERENCES}

1. Sharples, S. D., Clark, M., and Somekh, M. G. All-optical adaptive scanning acoustic microscope. Ultrasonics, 41:295-299, (2003).

2. Sharples, S. D., Clark, M., and Somekh, M. G. Surface acoustic wavefront sensor using custom optics. Ultrasonics, 42:647-651, (2004).

3. Sharples, S. D., Clark, M., and Somekh, M. G. Dynamic higher-order correction of acoustic aberration due to material microstructure. Applied Physics Letters, 81(12):22882290, (2002).

4. Goodman, J. W. Introduction to Fourier Optics. McGraw-Hill, 1968. 